\title{
Editorial
}

\section{Visual approaches for strengthening research, science communication and public health impact}

Geospatial Health was launched as a forum for the publication of essential epidemiological information derived from the application of geographical information systems (GIS), global positioning systems (GPS), spatial statistics and remote sensing from Earth-orbiting satellites. The above-mentioned tools were developed in parallel over the last 50 years and were originally used for diverse applications with little in common. For example, GIS evolved as an extension of map-making, while remote sensing and GPS both emanate from rocket and satellite technology. The swerve that brought these disciplines together was neither shared goals nor cross-sectional appeal but the unrelated, parallel scientific advances resulting in the personal computer and the Internet. Indeed, the first GIS packages required mainframe computing, but increased computing power and plummeting hardware prices helped the Environmental Systems Research Institute (ESRI) emerge and sustain its leading role in GIS software. The release of ESRI's first desktop mapping system with a graphical user interface (ArcView) in 1992 marked the start of the process that eventually made GIS sufficiently user-friendly to allow use by individual researchers without computer expertise.

Remote sensing was primarily intended for military intelligence, but the technology rapidly became the sine qua non for meteorology and cartography. It took, however, a surprisingly long time until its obvious possibilities for the medical and veterinary fields were realized. While GIS dramatically increases the amount of information that can be contained and manipulated in a map, remote sensing adds information on the landscape, e.g. surface temperature, humidity, rainfall, elevation, land cover, vegetation index etc., all parameters of direct importance for epidemiological research. Besides providing increasingly accurate measures for these parameters for local settings around the world, the inherent revisit aspect of the satellites supplying the imagery adds a nontrivial, temporal aspect to data collection. Although the development of supporting computer hard- and software, has grown exponentially, epidemiological research papers did not appear regularly until the late 1980s. Today, however, these technologies are well-known tools of the trade and few scientists working in the field of public and global health can manage without them. We, and all those participating in the editorial work for Geospatial Health, take pride in being part of the activities that have resulted in mounting reliance on cartographic representation of the burden of communicable diseases, including spatial data on the vectors and intermediate hosts of parasitic infections. In parallel with the powerful developments in the field of descriptive epidemiology, analytical approaches have gained importance owing to the growing need for surveillance due to the current, rapid environmental changes (land use and climate change), and population dynamics (modern and traditional migration). Graphically shown predictions serve priority setting, resource allocation and direct public health action as reflected in the increasing number of sound, analytical papers and focused case studies. In addition, the development of real-time surveillance systems, firmly based on GIS, GPS and remote sensing, facilitates the establishment of accurate, up-to-date early-warning systems (EWS), which are more and more needed in a world where an infectious disease can cross large distances in as little time as it takes a passenger aeroplane to circle the Earth.

The Internet has undeniably played an integral part in giving scientists and decision-makers access to near realtime data. The presentation of data based on interactive, computer-generated map applications represents a straightforward way of visualizing large numbers of datasets in a geographical context. This is part of the argument by Todd Presner in a 2010 discourse (http://cnx.org/content/m34246/1.6/), where he discusses endeavours cutting across a multitude of disciplines and relying on new forms of visual, cartographic and spatio-temporal narratives. The new ideas about publication models based on hybrid methodologies derive from the idea that visualization informs in a way that the written text alone cannot, empowering a broad range of people to both understand and mentally process voluminous amounts of material in a relatively short time. Thus, entering the 
new millennium, the old adage that a picture is worth more than a thousand words confirms that publication in this field should indeed have a strong graphic/visual bias.

Geospatial Health has previously pioneered the use of interactive illustrations and strengthens now this approach by launching a section for visual presentations, i.e. vHealth. Here, the text is truncated to the bare minimum, and complementary information supplied by means of a short video clip. Thus, visualization becomes an integral part of the scientific communication with the video showing relationships, objects, phenomena and even complex ideas in an easily understood form. Geospatial Health welcomes papers including such video clips aiming at the presentation of concepts, methodologies and results in a manner that is both entertaining and coherent. Each vHealth communication consists of abstract (indexed in PubMed and other databases), 3-5 keywords and short background information followed by a concise outlook paragraph with the key message. An Internet link providing an instructive video clip forms the innovative part, while boxes describe the purpose of the communication and the software used (limits given in 'Instruction for Authors'). The vHealth approach does not aspire to replace normal-length articles, but we believe that the traditional transmission of information will improve when complemented with a contextual mode envisioning presentations in a way that can be understood by all stakeholders. For example, the Health Resources Allocation Model (HRAM) by Maire et al. (2012), one of the presentations in the vHealth section inaugurated in this issue, provides a novel approach for teaching and training of health planners and health systems specialists. It is so far the only comprehensive tool for rational, sub-national health planning under resource constraints and the generation of relevant health systems research in a given social-ecological setting. Another two contributions, (Krieger et al., 2012 and Winkler et al., 2012), featured in the new vHealth section, demonstrate how key findings of complex entities, e.g. health impact assessment (HIA), can be visualized in brief video clips and indeed provide additional content that improves overall comprehension. Apart from catering to the academic community, it is felt that the visual approach facilitates communication with the wide variety of other stakeholders involved in research today, e.g. government offices, funding agencies, corporate upper-level management as well as the very people who live and work in the endemic areas. Clearly, the vHealth-section contributions can also become an accelerator for translational research resulting in public health actions by targeting and involving decision-makers and diseases programme managers.

We sincerely hope that you will enjoy the new section to Geospatial Health that is anticipated to stimulate exploration of communicating research findings not only to a scientific audience, but also to the broader public. We strive to emphasize health discussions assisting policy dialogue, strategy formulation and implementation within our overall goal of contributing to health development and well-being, particularly with respect to the most neglected diseases among the most neglected peoples of our world.

\section{References}

Krieger GR, Bouchard MA, Marques de Sa I, Paris I, Balge Z, Williams D, Singer BH, Winkler MS, Utzinger J, 2012. Enhancing impact: visualization as an integrated impact assessment strategy. Geospat Health 6, 303-306.

Maire N, Hegnauer M, Nguyen D, Godelmann L, Peterhans B, Hoffmann A, de Savigny D, Tanner M, 2012. A Health Resources Allocation Model (HRAM) for the 21st century. Geospat Health 6, 295-298.

Winkler MS, Krieger GR, Divall MJ, Singer BH, Utzinger J, 2012. Health impact assessment of industrial development projects: a spatio-temporal visualization. Geospat Health 6, 299-301.

Robert Bergquist

Editor-in-Chief

Marcel Tanner

Chair, Epidemiology and Medical Parasitology, University of Basel Director, Swiss Tropical and Public Health Institute (Swiss TPH) 